\title{
Uniportal video-assisted thoracoscopic surgery: safety, efficacy and learning curve during the first 250 cases in Quebec, Canada
}

\author{
Gabrielle Drevet, Paula Ugalde Figueroa \\ Division of Thoracic Surgery, Institut Universitaire de Pneumologie et Cardiologie de Quebec, Canada \\ Correspondence to: Paula Ugalde Figueroa, MD. Division of Thoracic Surgery, Institut Universitaire de Cardiologie et de Pneumologie de Québec, \\ 2725, chemin Sainte-Foy, Pavillon Laval, 3e étage, porte L-3540, Québec (Québec) G1V 4G5, Canada. Email: Paula.ugalde@criucpq.ulaval.ca.
}

\begin{abstract}
Background: Video-assisted thoracoscopic surgery (VATS) using a single incision (uniportal) may result in better pain control, earlier mobilization and shorter hospital stays. Here, we review the safety and efficiency of our initial experience with uniportal VATS and evaluate our learning curve.

Methods: We conducted a retrospective review of uniportal VATS using a prospectively maintained departmental database and analyzed patients who had undergone a lung anatomic resection separately from patients who underwent other resections. To assess the learning curve, we compared the first 10 months of the study period with the second 10 months.
\end{abstract}

Results: From January 2014 to August 2015, 250 patients underwent intended uniportal VATS, including 180 lung anatomic resections (72\%) and 70 other resections (28\%). Lung anatomic resection was successfully completed using uniportal VATS in 153 patients (85\%), which comprised all the anatomic segmentectomies (29 patients), $80 \%$ (4 of 5) of the pneumonectomies and 82\% (120 of 146) of the lobectomies attempted. The majority of lung anatomic resections that required conversion to thoracotomy occurred in the first half of our study period. Seventy patients underwent other uniportal VATS resections. Wedge resections were the most common of these procedures (25 patients, 35.7\%). Although 24 of the 70 patients (34\%) required the placement of additional ports, none required conversion to thoracotomy.

Conclusions: Uniportal VATS was safe and feasible for both standard and complex pulmonary resections. However, when used for pulmonary anatomic resections, uniportal VATS entails a steep learning curve.

Keywords: Video-assisted thoracoscopic surgery (VATS); uniportal; minimally invasive surgery; lobectomy

Submitted Dec 12, 2015. Accepted for publication Feb 03, 2016.

doi: $10.21037 /$ acs.2016.03.05

View this article at: http://dx.doi.org/10.21037/acs.2016.03.05

\section{Introduction}

Over the past 20 to 25 years, video-assisted thoracoscopic surgery (VATS) has evolved from a procedure primarily carried out for diagnostic purposes to one currently performed with therapeutic intent, even in complex cases. Indeed, VATS is now accepted as a feasible, safe and effective approach for the treatment of many intrathoracic pathological processes. In fact, thoracoscopic surgery is the preferred approach for lung anatomic resection in current practice guidelines for the treatment of early-stage lung cancer $(1,2)$ as well as for most benign intrathoracic pathologies. Benefits include decreased blood loss, decreased pain, shorter length of hospital stay and fewer complications (3-5).

In the early 1990s, VATS operations were done through 3-4 surgical ports but gradually surgeons began to use only 2 ports for many surgeries: one for the camera and one as a working incision for insertion of all other endoscopic tools (4,6-8). In 2004, the uniportal or single-port approach was introduced, initially for wedge pulmonary resection (9) and subsequently in 2010 for lobectomy (10). Uniportal VATS is quickly gaining popularity as a surgical approach and has been rapidly adopted across Europe and Asia (11-14). In a recently published propensity-matched analysis, uniportal VATS lobectomy was shown to be equivalent to multiport 
thoracoscopic lobectomy regarding most perioperative outcomes with the possible added advantages of better pain control and earlier patient mobilization (15). Likewise, decreased duration of chest tube drainage and shorter hospital stay after uniportal thoracic surgery have also been shown in some studies $(16,17)$.

In 2014, we began using a uniportal thoracoscopic strategy for the surgical treatment of most intrathoracic disorders. This study assesses the safety and efficiency of our initial experience with the uniportal VATS approach and evaluates our learning curve.

\section{Methods and materials}

\section{Patients}

We conducted a retrospective review of a prospectively maintained departmental database of all thoracic procedures performed at the Institut Universitaire de Cardiologie et de Pneumologie de Quebec, a tertiary referral center and teaching hospital. The database was queried for all surgical procedures performed by the senior author (PAU) between January 2014 and August 2015, because she was the only surgeon in the department performing uniportal VATS during the study period. Uniportal VATS was not attempted for resection of thymic or esophageal neoplasms. Demographic, histopathologic, surgical, perioperative and postoperative variables were analyzed. The patients were divided into two groups for analysis: one group included only patients who had undergone a lung anatomic resection via uniportal VATS and the other group included patients who had undergone other procedures using uniportal VATS including pulmonary wedge resection and pulmonary biopsy. To assess the learning curve, we compared the first 10 months of the study period (January 2014-October 2014) with the following 10 months (November 2014August 2015). The Ethics Committee of our institution approved this study and waived the requirement for informed consent.

\section{Surgical technique}

We performed all uniportal VATS procedures with the patient under general anesthesia and with single-lung ventilation. In most cases, we positioned the patient in full lateral decubitus position with flexion of the table at the level of the mid-chest, allowing slight widening of the intercostal space that improved exposure and facilitated access to the pleural cavity. A 3-4 $\mathrm{cm}$ incision was made in the fifth intercostal space anterior to the axillary line. A SurgiSleeve ${ }^{\mathrm{TM}}$ wound protector (Covidien-Medtronic, Minneapolis, MN, USA) was commonly placed and a 5-mm camera and all endoscopic tools were introduced into the pleural cavity through this incision. At the end of the procedure, a 5-mm skin incision was made and a chest tube was tunneled towards the working intercostal space. This is a modification of the original technique (10) which uses a drain inserted through the single-port incision.

\section{Statistical analysis}

Categorical variables are reported as $\mathrm{n}(\%)$. Continuous variables are expressed as a mean $\pm \mathrm{SD}$ or median [interquartile range (IQR), $25^{\text {th }}$ to $75^{\text {th }}$ percentile] depending upon variable distribution. Analyses were conducted using the statistical package SAS, version 9.4 (SAS Institute Inc., Cary, NC, USA).

\section{Results}

\section{Demographic data}

From January 2014 to August 2015, 250 patients underwent intended uniportal VATS at our institution. Mean age of the 141 men $(56.4 \%)$ and 109 women $(43.6 \%)$ was $63 \pm 11$ years (Table 1). The most frequent primary diagnosis was nonsmall cell lung cancer (NSCLC). Of the 250 patients, 180 patients $(72 \%)$ underwent lung anatomic resections and 70 patients $(28 \%)$ underwent uniportal VATS for other resections (Table 2).

\section{Uniportal VATS for lung anatomic resection}

Of the 180 patients who underwent a lung anatomic resection using uniportal VATS, 162 patients (90\%) had a final diagnosis of NSCLC (Table 1). The majority of patients $(72.2 \%)$ had a $30 \mathrm{~mm}$ or smaller pulmonary nodule and only 13 patients (7.2\%) had tumors larger than $50 \mathrm{~mm}$ (Table 1). Most of the lung anatomic resections were lobectomies (146 patients, $81.1 \%$ ) including 11 bilobectomies (Table 2). Sixteen of the lobectomy patients $(11.0 \%)$ underwent more complex surgical procedures including lobectomy with chest wall resection (3 patients), sleeve lobectomy (3 patients), lobectomy after induction therapy ( 2 patients), lobectomy with a tumor $>6 \mathrm{~cm}(6$ patients) and intrapericardial lobectomy (2 patients). 


\begin{tabular}{|c|c|}
\hline Characteristic & $\mathrm{N}(\%)^{1}$ \\
\hline Age (in years, mean \pm SD) & $63 \pm 10.9$ \\
\hline \multicolumn{2}{|l|}{ Sex } \\
\hline Male & $141(56.4)$ \\
\hline Female & $109(43.6)$ \\
\hline Diagnosis (all patients) & $N=250$ \\
\hline \multicolumn{2}{|l|}{ Cancer } \\
\hline NSCLC & $173(69.2)$ \\
\hline Secondary lung cancer & $8(3.2)$ \\
\hline Other & $14(5.6)$ \\
\hline \multicolumn{2}{|l|}{ Benign } \\
\hline Pneumothorax & $6(2.4)$ \\
\hline Empyema & $9(3.6)$ \\
\hline Pleural effusion & $3(1.2)$ \\
\hline Other & $37(14.8)$ \\
\hline $\begin{array}{l}\text { Diagnosis (patients who underwent } \\
\text { lung anatomic resection) }\end{array}$ & $N=180$ \\
\hline \multicolumn{2}{|l|}{ Cancer } \\
\hline NSCLC & $162(90.0)$ \\
\hline SCLC & $9(5.0)$ \\
\hline Secondary lung cancer & $6(3.3)$ \\
\hline Benign & $11(6.1)$ \\
\hline $\begin{array}{l}\text { Nodule size (patients who underwent } \\
\text { lung anatomic resection) }\end{array}$ & $\mathrm{N}=180$ \\
\hline $5-30 \mathrm{~mm}$ & $130(72.2)$ \\
\hline $31-50 \mathrm{~mm}$ & $32(17.8)$ \\
\hline$\geq 51 \mathrm{~mm}$ & $13(7.2)$ \\
\hline Non-nodular & $5(2.8)$ \\
\hline
\end{tabular}

${ }^{1}$, Unless otherwise noted. NSCLC, non-small cell lung cancer; SCLC, small cell lung cancer; SD, standard deviation.

Anatomic lung resection was successfully performed with the uniportal approach in 153 patients $(85 \%)$. The anatomic resection was completed using only the uniportal approach in all of the anatomic segmentectomies (29 patients) and most of the pneumonectomies (4 of 5 patients). Of the 146 attempted uniportal VATS lobectomies, the procedure could not be completed using the uniportal approach in 26 patients (17.8\% of lobectomies attempted) (Table 2).

When lung anatomic resection could not be completed using a uniportal approach, a minimally invasive approach was still possible in 16 of the 27 patients (59.2\%) (Table 3). This was accomplished through VATS using an additional

\begin{tabular}{|c|c|c|c|}
\hline Surgery & $\mathrm{N}(\%)$ & $\begin{array}{l}\text { Uniportal } \\
(\%)\end{array}$ & $\begin{array}{l}\text { Non- } \\
\text { uniportal } \\
\text { (\%) }\end{array}$ \\
\hline $\begin{array}{l}\text { Lung anatomic } \\
\text { resections }\end{array}$ & $\mathrm{N}=180$ & $153(85)$ & $27(15.0)$ \\
\hline Lobectomy & $146(81.1)$ & 120 (82.2) & $26(17.8)$ \\
\hline Segmentectomy & $29(16.1)$ & $29(100.0)$ & 0 \\
\hline Pneumonectomy & $5(2.8)$ & $4(80.0)$ & $1(20.0)$ \\
\hline $\begin{array}{l}\text { Other thoracic } \\
\text { surgeries }\end{array}$ & $\mathrm{N}=70$ & $46(66.0)$ & $24(34.0)$ \\
\hline Wedge resection & $25(35.7)$ & 25 (100.0) & 0 \\
\hline Decortication & $15(21.4)$ & $8(53.0)$ & $7(47.0)$ \\
\hline Bullectomy & $8(11.4)$ & $6(75.0)$ & $2(25.0)$ \\
\hline $\begin{array}{l}\text { Mediastinal mass } \\
\text { resection }\end{array}$ & $8(11.4)$ & $6(75.0)$ & $2(25.0)$ \\
\hline Lymph node biopsy & $6(8.6)$ & $6(100.0)$ & 0 \\
\hline Pleural biopsy & $3(4.3)$ & $3(100.0)$ & 0 \\
\hline Other & $5(7.1)$ & $5(100.0)$ & 0 \\
\hline
\end{tabular}

Table 3 Approach for lung anatomic resection instead of uniportal VATS

\begin{tabular}{ll}
\hline & $\mathrm{N}(\%)$ \\
\hline Surgery used & $13(7.2)$ \\
2-port VATS & $3(1.6)$ \\
Hybrid procedure & $11(6.1)$ \\
Thoracotomy & \\
Reason for thoracotomy & $7(3.9)$ \\
Technical difficulties & $1(0.6)$ \\
Sleeve & $2(1.1)$ \\
Arterioplasty & $3(1.6)$ \\
Tumor size & $1(0.6)$ \\
Vascular malformation & $4(2.2)$ \\
Vascular accident & VATS, video-assisted thoracoscopic surgery.
\end{tabular}

port in 13 patients. The technical difficulty of stapling the superior pulmonary vein was the primary reason to add a port in 11 of the 13 patients. Conversion to a hybrid procedure for chest wall invasion (uniportal VATS and a mini-thoracotomy) was necessary in 3 patients. Conversion to thoracotomy was necessary in 11 patients $(6.1 \%)$. Yet, in 7 of the patients $(3.9 \%)$, the conversion to thoracotomy 


\begin{tabular}{|c|c|c|}
\hline Complication $^{1}$ & $\begin{array}{l}\text { Uniportal lung } \\
\text { anatomic } \\
\text { resections } \\
\mathrm{N}=180(\%)\end{array}$ & $\begin{array}{l}\text { Other uniportal } \\
\text { thoracic } \\
\text { surgeries } \\
\mathrm{N}=70(\%)\end{array}$ \\
\hline \multicolumn{3}{|l|}{ Minor complications } \\
\hline Prolonged air leak & $26(14.4)$ & $4(5.7)$ \\
\hline Heart rhythm disorders & $18(10.0)$ & $6(8.6)$ \\
\hline Pulmonary tract infection & $4(2.2)$ & $1(1.4)$ \\
\hline $\begin{array}{l}\text { Temporary laryngeal } \\
\text { nerve paralysis }\end{array}$ & $2(1.1)$ & 0 \\
\hline \multicolumn{3}{|l|}{ Major complications } \\
\hline ARDS & $2(1.1)$ & $1(1.4)$ \\
\hline Pneumonia requiring ICU & 0 & $2(2.9)$ \\
\hline Bronchopleural fistulae & $3(1.7)$ & 0 \\
\hline Chylothorax & $1(0.6)^{2}$ & $1(1.4)^{2}$ \\
\hline Hemothorax & $1(0.6)$ & $1(1.4)^{2}$ \\
\hline Cardiogenic shock & $2(1.1)$ & 0 \\
\hline \multicolumn{3}{|c|}{$\begin{array}{l}{ }^{1} \text {, Some patients experienced more than } 1 \text { complication; } \\
{ }^{2} \text {, Surgical repair was required. ARDS, acute respiratory } \\
\text { distress syndrome; ICU, intensive care unit. }\end{array}$} \\
\hline
\end{tabular}

was not considered a failure due to the clinical complexity of the procedure. The remaining 4 thoracotomies $(2.2 \%)$ were necessitated by vascular accidents causing injury to the pulmonary artery (Table 3).

The median operating time (incision to skin closure) for lung anatomic resection with intended uniportal VATS was 144 minutes (range, 60-600 minutes; IQR, 120-180 minutes) with a median blood loss of $70 \mathrm{~mL}$ (IQR, $40-150 \mathrm{~mL}$ ). In the 4 cases of vascular accident, the median blood loss was $1,700 \mathrm{~mL}$ (IQR, $250-3,250 \mathrm{~mL}$ ). The median length of hospitalization for patients who underwent lung anatomic resection with an intended uniportal VATS approach was 4 days (IQR, 3-5 days).

Complications occurred in 53 patients (29.4\%) (Table 4). Major complications occurred in 9 patients (5\%) and included 3 cases $(1.7 \%)$ of bronchial fistula: one in a patient who received chemoradiation prior to uniportal VATS, one in a frail patient who underwent a sleeve lobectomy because he could not tolerate a pneumonectomy and one in a patient who underwent a lower lobectomy (Table 4). Among the 180 patients who underwent intended uniportal lung anatomic resection, there was no operative mortality and 1 perioperative mortality $(0.6 \%)$ that was secondary to acute respiratory distress syndrome 13 days postoperatively in the same frail patient who developed a bronchopleural fistula after right upper lobe sleeve lobectomy.

\section{Uniportal VATS for other procedures}

During the study period, 70 patients underwent resections other than lung anatomic resection using an intended uniportal VATS approach. Wedge resections were the most common procedure and were performed in 25 patients (35.7\%). Uniportal VATS was also used for decortication for empyema, bullectomy, mediastinal mass resection and lymph node staging (Table 2). Although approximately $1 / 3$ of the patients (24 patients, 34\%) required the placement of additional ports, none required conversion to thoracotomy. Of the 24 patients, 20 (28.6\%) required one additional port and $4(5.7 \%)$ needed two additional ports to successfully complete the VATS procedure. All of the procedures requiring additional ports were decortication, bullectomy or mediastinal mass resection (Table 2).

The median operative time for patients who underwent intended uniportal VATS for indications other than lung anatomic resection was 84 minutes (range, 60-108 minutes) with a median blood loss of $20 \mathrm{~mL}$ (IQR, 10-100 mL). The median length of hospital stay was 3 days (IQR, 2-6 days). Complications occurred in 12 patients (17.14\%). The most frequent complications were prolonged air leak (4 patients, $5.7 \%$ ) and heart rhythm disorder (6 patients, 8.6\%) (Table 4). Although chylothorax and hemothorax occurred in only one patient apiece, both necessitated re-intervention. There was no operative or perioperative mortality among the patients who underwent resection via uniportal VATS for procedures other than lung anatomic resection.

\section{Evaluation of the learning curve for uniportal VATS}

To evaluate our learning curve, we compared the success rate of performing uniportal lung anatomic resection and other uniportal VATS resection in the first 10 months and last 10 months of the study period. For this analysis, the 3 patients who underwent combined lobectomy and chest wall resection were excluded. In the uniportal lung anatomic resection group, 95 surgeries (53\%) were performed in the first half of the study and 85 surgeries $(47 \%)$ were performed in the in the second half. Of the 24 lung anatomic resections that could not be completed using a uniportal approach, 16 (66.7\%) occurred during the first half of the study including all cases of thoracotomy as a result of a vascular accident, 


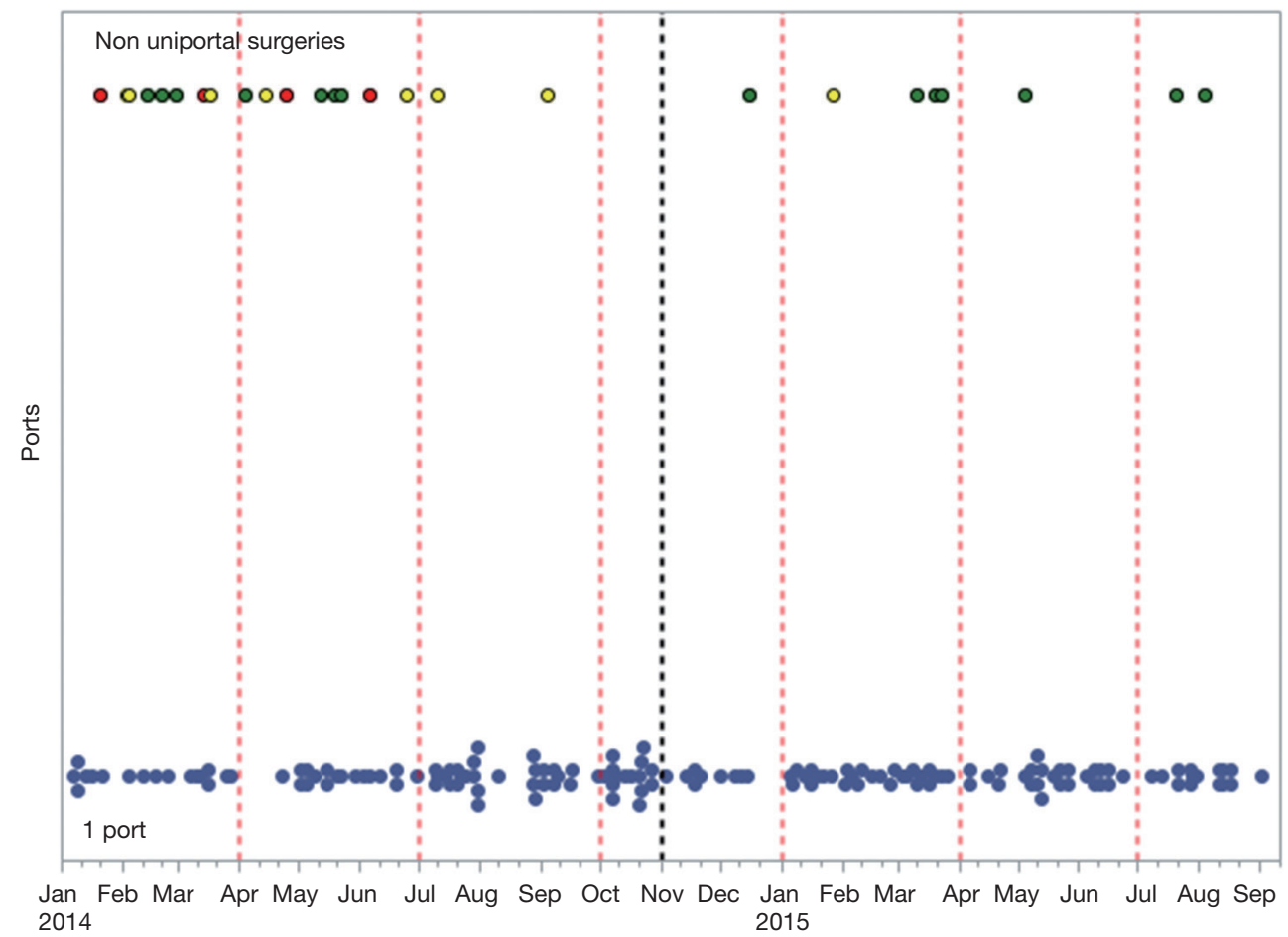

Figure 1 Learning curve for lung anatomic resection using uniportal VATS. Blue, uniportal VATS (155 cases); green, VATS requiring 2 ports (14 cases); red, thoracotomy for vascular accident (4 cases); yellow, thoracotomy for technical difficulties (7 cases). VATS, video-assisted thoracoscopic surgery.

most cases of thoracotomy due to technical difficulties (6 of 7 cases, $85.7 \%$ ) and about half of the cases requiring placement of an additional port (6 of 13 cases, 46.2\%) (Figure 1). In the patients who underwent uniportal VATS procedures other than lung anatomic resection, 36 surgeries (51.4\%) were performed in the first half of the study and 34 surgeries (48.6\%) in the second half. Additional ports were necessary in 13 cases in the first half of the study $(36.1 \%)$ and 11 cases in the second half (32.4\%).

\section{Discussion}

In this study, we describe our series of 250 patients who required surgery for thoracic pathologies and were treated with intended uniportal VATS resection. Due to the diversity of the surgeries, we analyzed the patients' outcomes based on whether uniportal VATS was used for a lung anatomic resection or for any other thoracic resection. When utilizing uniportal VATS for lung anatomic resection, we placed an additional port in approximately $7 \%$ of patients and converted to a thoracotomy in approximately
$6 \%$ of patients. When attempting VATS resection other than lung anatomic resection using the uniportal approach, we placed addition ports in about one third of the surgeries, but did not convert to a thoracotomy or hybrid procedure in any patient. Most postoperative complications were minor. The learning curve for uniportal lung anatomic resection was steep as evidenced by the fact that the majority of conversions to thoracotomy, multiport VATS or a hybrid procedure occurred in the first half of our study period.

In our center, all lung resections are started using VATS, so a higher conversion rate to thoracotomy is expected in complex cases than may be seen in other centers. This may also explain the range of postoperative complications observed. In this series, approximately $10 \%$ of the patients who underwent an attempted uniportal lung anatomic resection were complex cases. For some of these patients, one could even argue that VATS was not indicated due to the difficulty of the case. Because technical difficulties are expected in complex cases, thoracotomy should be considered a prophylactic conversion rather than a failure of an intended minimally invasive approach in some 
patients. We find it hard to compare our conversion rate with published VATS series since most series have focused on early-stage lung cancer, excluded pneumonectomies and examined a more homogenous group of patients than this series $(4,18)$. A preliminary analysis suggested that if only patients undergoing lobectomy or segmentectomy for early-stage lung cancer were analyzed, our conversions to thoracotomy would be solely due to 2 cases of vascular accidents. The complications that we observed in patients who underwent lung anatomic resections using uniportal VATS were similar to those reported in other studies $(4,8)$ and included prolonged air leak, heart rhythm disorders and pulmonary infections. Major complications, such as acute respiratory distress syndrome and cardiogenic shock, have also been described and are not particularly predominant after uniportal VATS (9). Moreover, they are related to the patient's preoperative clinical condition and the type of lung resection performed, as supported by our series.

In the series, we encountered four cases where thoracotomy was required secondary to vascular injury: two cases that occurred during lobectomy for early-stage lung cancer, one case during a lobectomy for a central mass larger than $6 \mathrm{~cm}$ and one case during a pneumonectomy for a central tumor invading the pulmonary artery. The median blood loss for the entire group was low, however when a vascular accident occurred median blood loss increased exponentially (from 70 to $1,700 \mathrm{~mL}$ ). The correct assessment of any bleeding is of paramount importance during VATS lung anatomic resection. Inadequate management of the bleeding source may result in massive hemorrhage due to the limited space to work. With experience, the rate of technical complications diminishes and bleeding can often be controlled thoracoscopically.

When we analyzed our learning curve over the 20-month study period, the success rate of uniportal procedures, particularly uniportal lung anatomic resections, increased over time. In fact, all vascular accidents occurred early in our experience. The need to place a second port to staple the superior pulmonary vein also decreased with time. It is clear that with growing experience, the success rate of performing complex lung surgery using uniportal VATS improves. Unfortunately, publications on conversion to thoracotomy, placement of an additional port or complications as a function of experience during the adoption of the uniportal approach for lung resection are scarce $(5,16)$, which restrains comparisons that can be made with our series.

All of the surgeries in this series were performed by a single surgeon with fellowship training in minimally invasive thoracic surgery. Although all patients were treated at a tertiary referral center that is also a teaching hospital, the series suggests that uniportal VATS could be adopted in a variety of practice settings as long as the surgeon adopting the approach has sufficient experience with standard multiport VATS procedures. The fact that a single surgeon performed all the procedures is also a limitation of the study, as the effects of the surgeon's experience on the learning curve cannot be ascertained. Other limitations include those inherent to all retrospective series.

\section{Conclusions}

In our series, uniportal VATS was safe and feasible. This is an emerging technique increasingly used for standard and complex pulmonary resections. However, when used for lung anatomic resection, uniportal VATS entails a steep learning curve.

\section{Acknowledgements}

The authors thank Serge Simard at our institution for performing the statistical analysis. They also thank Shannon Wyszomierski for her editorial assistance.

\section{Footnote}

Conflicts of Interest: The authors have no conflicts of interest to declare.

\section{References}

1. Ettinger DS, Wood DE, Akerley W, et al. Non-Small Cell Lung Cancer, Version 6.2015. J Natl Compr Canc Netw $2015 ; 13: 515-24$.

2. Howington JA, Blum MG, Chang AC, et al. Treatment of stage I and II non-small cell lung cancer: Diagnosis and management of lung cancer, 3rd ed: American College of Chest Physicians evidence-based clinical practice guidelines. Chest 2013;143:e278S-313S.

3. Scott WJ, Allen MS, Darling G, et al. Video-assisted thoracic surgery versus open lobectomy for lung cancer: a secondary analysis of data from the American College of Surgeons Oncology Group Z0030 randomized clinical trial. J Thorac Cardiovasc Surg 2010;139:976-81; discussion 981-3.

4. Onaitis MW, Petersen RP, Balderson SS, et al. 
Thoracoscopic lobectomy is a safe and versatile procedure: experience with 500 consecutive patients. Ann Surg 2006;244:420-5.

5. Paul S, Altorki NK, Sheng S, et al. Thoracoscopic lobectomy is associated with lower morbidity than open lobectomy: a propensity-matched analysis from the STS database. J Thorac Cardiovasc Surg 2010;139:366-78.

6. McKenna RJ Jr. Lobectomy by video-assisted thoracic surgery with mediastinal node sampling for lung cancer. J Thorac Cardiovasc Surg 1994;107:879-81; discussion 881-2.

7. Roviaro G, Varoli F, Rebuffat C, et al. Major pulmonary resections: pneumonectomies and lobectomies. Ann Thorac Surg 1993;56:779-83.

8. Yim AP, Izzat MB, Liu HP, et al. Thoracoscopic major lung resections: an Asian perspective. Semin Thorac Cardiovasc Surg 1998;10:326-31.

9. Rocco G, Martin-Ucar A, Passera E. Uniportal VATS wedge pulmonary resections. Ann Thorac Surg 2004;77:726-8.

10. Gonzalez D, Paradela M, Garcia J, et al. Single-port videoassisted thoracoscopic lobectomy. Interact Cardiovasc Thorac Surg 2011;12:514-5.

11. Gonzalez-Rivas D. VATS lobectomy: surgical evolution

Cite this article as: Drevet G, Ugalde Figueroa P. Uniportal video-assisted thoracoscopic surgery: safety, efficacy and learning curve during the first 250 cases in Quebec, Canada. Ann Cardiothorac Surg 2016;5(2):100-106. doi: 10.21037/ acs.2016.03.05 from conventional VATS to uniportal approach. ScientificWorldJournal 2012;2012:780842.

12. Ng CS. Uniportal VATS in Asia. J Thorac Dis 2013;5 Suppl 3:S221-5.

13. Pischik VG. Technical difficulties and extending the indications for VATS lobectomy. J Thorac Dis 2014;6:S623-30.

14. Ismail M, Helmig M, Swierzy M, et al. Uniportal VATS: the first German experience. J Thorac Dis 2014;6:S650-5.

15. Shen Y, Wang H, Feng M, et al. Single- versus multipleport thoracoscopic lobectomy for lung cancer: a propensity-matched study†. Eur J Cardiothorac Surg 2016;49 Suppl 1:i48-i53.

16. Li C, Ma H, He J, et al. Clinical analysis of thoracoscopic lobectomy in the treatment of peripheral lung cancer with single utility port. Zhongguo Fei Ai Za Zhi 2013;16:487-91.

17. Akter F, Routledge T, Toufektzian L, et al. In minor and major thoracic procedures is uniport superior to multiport video-assisted thoracoscopic surgery? Interact Cardiovasc Thorac Surg 2015;20:550-5.

18. McKenna RJ Jr, Houck W, Fuller CB. Video-assisted thoracic surgery lobectomy: experience with 1,100 cases. Ann Thorac Surg 2006;81:421-5; discussion 425-6. 